\title{
Natural Locomotion Interfaces - With a Little Bit of Magic!
}

\author{
Frank Steinicke \\ Immersive Media Group \\ Department of Computer Science \& Department of Human-Computer-Media \\ University of Würzburg \\ Email: frank.steinicke@uni-wuerzburg.de
}

\begin{abstract}
The mission of the Immersive Media Group (IMG) is to develop virtual locomotion user interfaces which allow humans to experience immersive virtual environments (IVEs) by means of natural walking. Traveling through IVEs by means of real walking is an important activity to increase naturalness of virtual reality (VR)-based interaction. However, the size of the virtual world often differs from the size of the tracked lab space so that a straightforward implementation of omni-directional and unlimited walking is not possible. Redirected walking is one concept to address this issue by inconspicuously guiding the user on a physical path that may differ from the path the user perceives in the virtual world.

In the scope of the LOCUI project we analyze how gains of locomotor speed, turns and curvatures can gradually alter the physical trajectory with respect to the path perceived in the virtual world without the users observing any discrepancy. Thus, users can be guided in order to avoid collisions with physical obstacles (e.g., lab walls) or they can be guided to arbitrary locations in the physical space. For example, if the user approaches a virtual object, he/she can be guided to a real proxy prop that is registered to and aligned with its virtual counterpart. Hence, the user can interact with a virtual object by touching the corresponding real-world proxy prop that provides haptic feedback. With such a user interface it becomes possible to intuitively interact with any virtual object by touching registered real-world props.
\end{abstract}

Index Terms-Virtual reality, immersive media, locomotion.

\section{INTRODUCTION}

In the real world we navigate with ease by walking, running, driving etc., but in the virtual world realistic simulation of these locomotion techniques is difficult to achieve. While moving in the real world, sensory information such as vestibular, proprioceptive, and efferent copy signals as well as visual information create consistent multi-sensory cues that indicate ones own acceleration, speed and direction of travel. In this context walking is the most basic and intuitive way of moving within the real world. Keeping such an active and dynamic ability to navigate through large-scale immersive virtual environments (IVEs) is of great interest for many 3D applications demanding locomotion, such as urban planning, tourism, 3D entertainment, serious games, robotics etc. Although the mentioned application domains are inherently three-dimensional and sometimes provide a good sense of locomotion by exclusive visual stimulation, usually VR-based user interfaces are not supported, least of all real walking is possible [1].

Mission of the Immersive Media Group: The mission of the Immersive Media Group (IMG) is to develop virtual locomotion user interfaces which allow humans to experience immersive virtual environments (IVEs) by means of the natural walking metaphor. IMG was founded in 2011 and is affiliated to the institute of computer science as well as the institute of human-computer-media. One of the labs major projects is the LOCUI project. The goal of this project is to provide means for the most natural way of navigating through such VEs, i.e., traveling by real walking.

\section{A. Locomotion in Virtual Environments}

VEs were initially restricted to visual displays, combined with interaction devices for providing (often unnatural) inputs (e. g. a joystick or mouse) to generate self-motion. More and more research groups are investigating natural, multimodal methods of generating self-motion. Typically, IVEs are characterized, for example, by head-mounted displays (HMDs) and a tracking system for measuring position and orientation data.

An obvious approach to implement real walking in such a setup is to map the users head movements or gaits to changes of the virtual camera by means of a one-to-one mapping. This technique has the drawback that the users movements are restricted by the limited range of the tracking sensors and a rather small tracked lab space in the real word. Therefore, concepts for virtual locomotion interfaces are needed that enable walking over large distances in the virtual world while physically remaining within a reasonably small space. To address unlimited walking in IVEs, various prototypes of interface devices have been developed to prevent a displacement in the real world. These devices include torus-shaped omnidirectional treadmills, motion foot pads, robot tiles and motion carpets [3]. All these systems are costly and support only a single user, for multi-walker scenarios it is necessary to equip each user with a separate device. Although these hardware systems represent enormous technological achievements, most likely they will not be generally available in the foreseeable future due to the described limitations. Hence there is a tremendous demand for alternative, more cost-effective and practical approaches. As a solution to this challenge, traveling by exploiting walk-like gestures has been proposed in many 


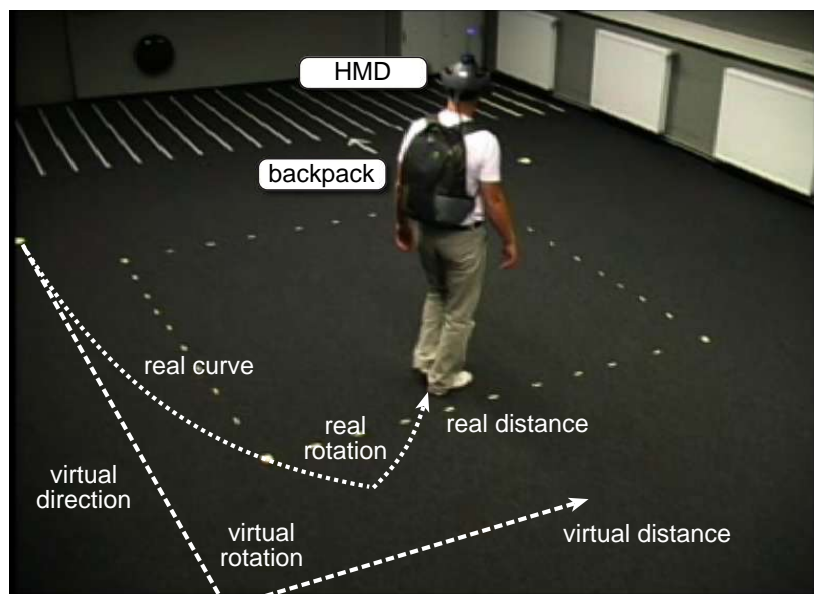

Fig. 1. Redirected walking scenario: a user walks in the real environment on a different path with a different length in comparison to the perceptual path in the virtual world.

different variants, giving the user the impression of walking. For example, the walking-in-place approach exploits walkinglike gestures to travel through a VE, while the user stays physically at almost the same position. However, real walking has been shown to be a more presence-enhancing and natural locomotion technique than any other navigation metaphor.

\section{B. Redirected Walking}

Redirected walking [5] is a promising solution to the problem of limited tracking space and to the challenge of providing users with the ability to explore an IVE by walking. The technique redirects users by manipulating the displayed scene, causing them to unknowingly compensate by repositioning or reorienting themselves. Different approaches to redirect a user in an IVE have been suggested. A common approach is to scale translational movements, for example, to cover a virtual distance that is larger than the distance walked in the physical space. With most reorientation techniques, the virtual world is imperceptibly rotated around the center of a stationary user until he/she is oriented such that no physical obstacles are in front of her [2]. Then, the user can continue to walk in the desired virtual direction. Reorientation can also be applied while the user walks. For instance, if the user wants to walk straight ahead for a long distance in the virtual world, small rotations of the camera redirect him/her to walk unconsciously on a circular arc in the opposite direction in the real world. When redirecting a user, the visual sensation is consistent with motion in the IVE, but vestibular- proprioceptive sensation reflects motion in the physical world. However, if the induced manipulations are small enough, humans do not perceive the discrepancies, and they have the impression of being able to walk in the virtual world in any direction without restrictions. Redirection techniques have been applied particularly in the field of robotics for controlling a remote robot by walking. With redirected walking it becomes possible to guide humans to arbitrary locations in the physical space, e.g., proxy props which provide passive haptic feedback. Passive haptic feed- back has been used effectively to support the natural sensation of touch. The main idea is to replicate counterparts of virtual objects such as walls and tables in the physical space and to arrange them correspondingly. Humans can interact with virtual objects by touching their real-world counterparts. It has been shown that this increases the presence in the IVE significantly. When redirected walking techniques are applied it becomes possible to change the mapping between virtual objects and proxy props such that a single proxy prop may represent multiple virtual objects. For example, a user can interact with several virtual tables by touching always the same proxy table in the real world. When the user approaches another virtual table, he/she is guided along a circle in the lab space bringing him/her back to the same proxy table. In Figure 1 the concepts of redirected walking are illustrated, the physical trajectory deviates from the path perceived in the virtual world.

\section{PSYCHOPHYSICAL EXPERIMENTS}

We have performed a series of experiments in order to identify thresholds which indicate the tolerable amount of deviation between vision and proprioception while walking [6].

Our results show that users can be turned physically about $49 \%$ more or $20 \%$ less than the perceived virtual rotation without noticing the difference. Our results agree with previous findings that users are more sensitive to scene motion if the scene moves against head rotation than if the scene moves with head rotation. Walked distances in the real world can be downscaled by $14 \%$ and up-scaled by $26 \%$, when they are mapped to virtual motions. This asymmetry coincides with previous findings that users tend to underestimate distances [2]. Subjects estimate that they have walked $5 \mathrm{~m}$ distance after waking only 4.69. When applying curvature gains users can be redirected such that they unknowingly walk on a circular arc when the radius is greater or equal to $22 \mathrm{~m}$.

Certainly, redirected walking is a subjective matter, but the results have potential to serve as thresholds for the development of future locomotion interfaces. The detection thresholds derived from our experiments are conservative estimates, since a subject's task was to detect discrepancies between vestibular, proprioceptive, as well as efferent copy signals perceived in the real world and visual feedback perceived in the virtual environment. In actual VR-based applications based on redirected walking users will not be confronted with such discrepancies in an obvious way, instead users will focus on other tasks such as selection or manipulation of objects in space. We have experienced that subjects tolerate substantially greater gains when they are not aware of the manipulation, in particular if they are engaged in their primary tasks.

\section{EXAMPLE PROJECTS}

In this section we will present two example projects, which have been evolved from the LOCUI project. Both projects address the challenge of long distance travel for which real walking is often inappropriate. 


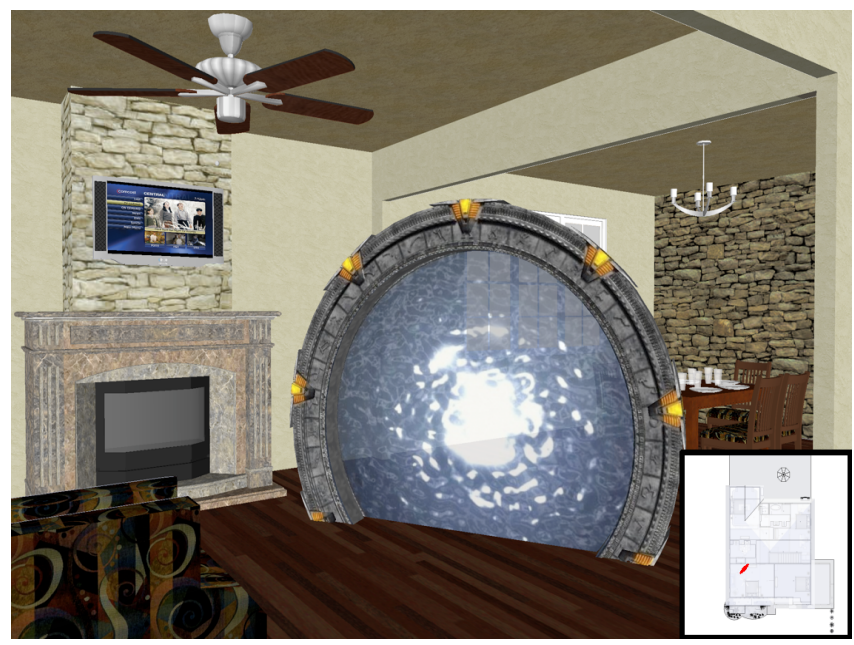

(a)

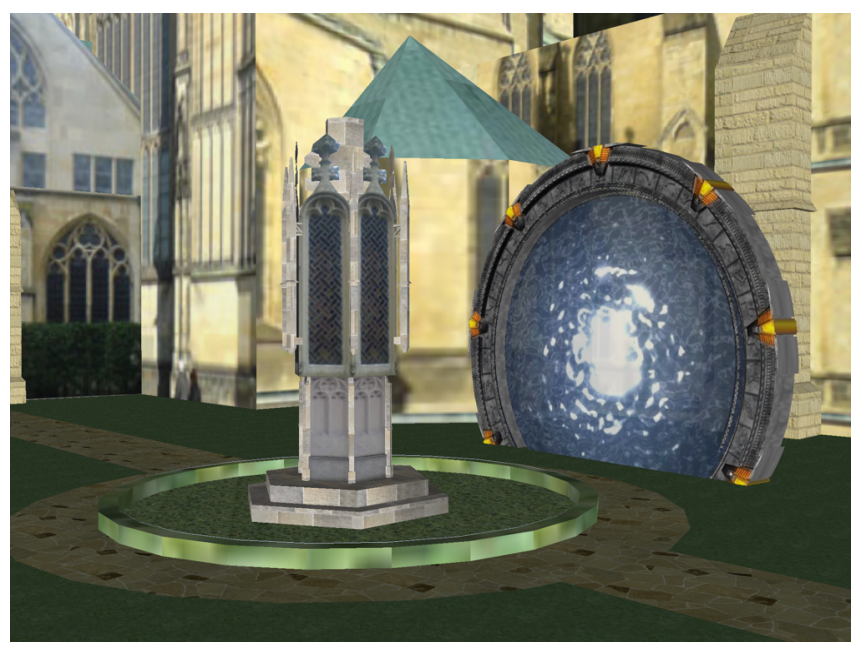

(b)

Fig. 2. Illustration of two connected portals: (a) portal displayed in a building with a $2 \mathrm{D}$ map of the environment, and (b) the target portal outside the building.

\section{A. Virtual Portals}

In many real-world situations we avoid to walk long distance, but rather prefer more efficient transport services. In the virtual world efficient traveling techniques may be implemented by means of magic metaphors.

In this context, virtual portals are three-dimensional doorways that connect one virtual location with another and can be entered by users in order to get to that place and back. Besides connecting different locations in the same VE, portals can also provide doorways between different VEs [2]. We use such portals as means for users to travel from one place in the virtual world to another place (see Figure 2). By walking through such a virtual portal or doorway (see Figure 2(a)), users can leave the current location and enter a previously selected place (see Figure 2(b)). Such selections can be performed on a two-dimensional map of the environment (see Figure 2(a)). The transition between two places is intuitive, since users just walk from one place through a doorway to another location. In order to allow an easy exploration of the $\mathrm{VE}$, we fade out the just crossed portal behind users after a few seconds.

\section{B. Jumper Metaphor}

We developed another magical metaphor to overcome large distances in the VE. In this context we developed the jumper metaphor, which combines real walking for short distances with an acceleration triggered, smooth jumping locomotion for large distances [1]. In a small range, users can explore objects by real walking and using small head movements while perceiving motion parallax and occlusion effects similar to the real world. To travel over large distances (i.e., at least 2 meters), the user has to specify the intended jump target by gazing at the target for some ms. Slight variations in the user's viewing direction are tolerated, since users generally move the head during the entire time. To give corresponding visual feedback, a visual target is projected to the target position according to the user's viewing direction and the face normal vector at the target position (see Fig. 3(a)). The visual target grows constantly to its full size. The user can choose a different jump target by gazing at a point outside the displayed target area. After the user has specified the jump target, he/she can initiate the jump to that target by exceeding an acceleration threshold during the movement towards the target. In order to avoid disorientation, we use a smooth easein/ease-out jump animation of the straight connection of the current user position and the jump target. The destination is adjusted in the direction towards the user's current position along the face normal at the target position and the terrain height difference in order to prevent jumping directly into an object (cf. Fig. 3(c)). We use a motion blur effect in the border of the viewport which fades out to the center (see Fig. 3(b)).

\section{CONCLUSION}

In this paper we introduced the Immersive Media Group and described one of our main projects - the LOCUI project. We presented how we develop virtual locomotion user interfaces which allow humans to experience arbitrary 3D environments by means of the natural walking enhanced by magic metaphors like portals or virtual jumping. The example projects illustrate the potential of such techniques, in particular for architecture, urban planning, tourism, 3D entertainment, or serious fullbody games.

Researchers and students from the domains of computer science, human-computer-interaction, media communication and psychology cooperate in the Immersive Media Group. Lectures of IMG give courses in computer science, computer science in media, human-computer-interaction, modeling, design, animation, virtual reality, games and edutainment. 

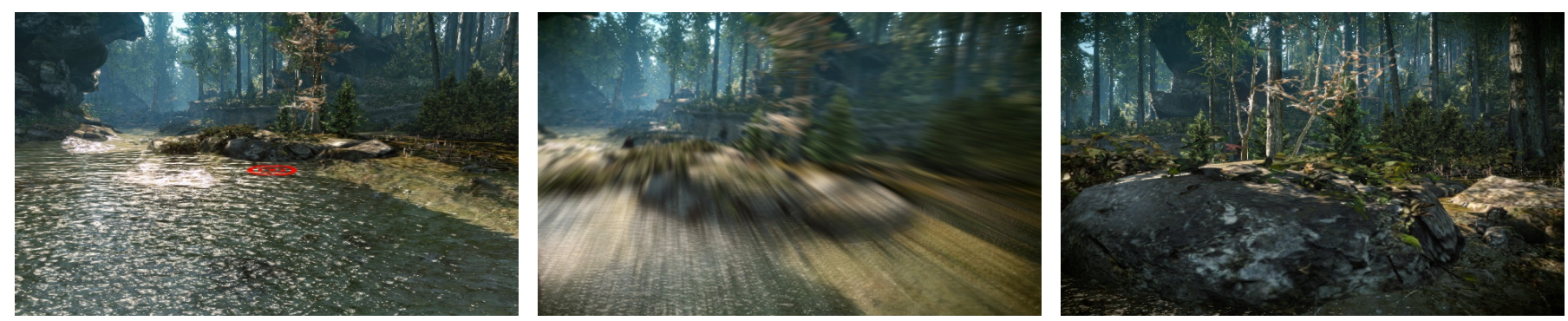

Fig. 3. Illustration of the jumper metaphor showing a jump sequence: (a) user's view to a target location before the jump, (b) frame during the jump, and (c) after the jump at the target location.

\section{ACKNOWLEDGMENT}

This work was partly supported by grants from the Deutsche Forschungsgemeinschaft (DFG) in the scope of the LOCUI project.

\section{REFERENCES}

[1] B. Bolte, G. Bruder, F. Steinicke: The Jumper Metaphor: An Effective Navigation Technique for Immersive Display Setups. Proceedings of Virtual Reality International Conference (VRIC). pp. 34-41, 2011

[2] G. Bruder, F. Steinicke, K. Hinrichs: Arch-Explore: A Natural User Interface for Immersive Architectural Walkthroughs. Proceedings of IEEE Symposium on 3D User Interfaces (3DUI). IEEE Press, pp. 75-82, 2009

[3] J.M. Hollerbach: Locomotion Interfaces, Handbook of Virtual Environments: Design, Implementation, and Applications, K.M. Stanney, ed., Lawrence Erlbaum Associates, Inc., pp. 239-254, 2002
[4] F. Steinicke, G. Bruder, S. Kuhl, P. Willemsen, M. Lappe, K. Hinrichs: Judgment of Natural Perspective Projections in Head-Mounted Display Environments. Proceedings of ACM Symposium on Virtual Reality Software and Technology (VRST). ACM Press, 2009

[5] F. Steinicke, G. Bruder, J. Jerald, H. Frenz, M. Lappe: Estimation of Detection Thresholds for Redirected Walking Techniques. In: IEEE Transactions on Visualization and Computer Graphics (16)1, pp. 17-27, 2010.

[6] F. Steinicke, G. Bruder, J. Jerald, H. Frenz, M. Lappe: Analyses of Human Sensitivity to Redirected Walking. ACM Symposium on Virtual Reality Software and Technology (VRST). ACM Press, pp. 149-156, 2008.

[7] F. Steinicke, G. Bruder, K. Hinrichs, A. Steed: Presence-Enhancing Real Walking User Interface for First-Person Video Games. ACM SIGGRAPH Games Paper, ACM Press, 2009. 